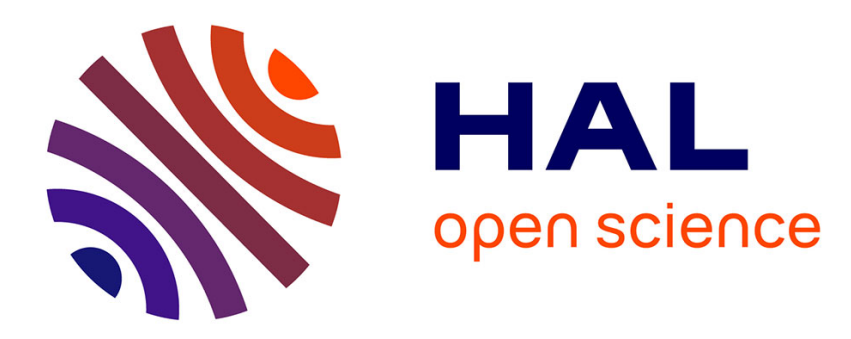

\title{
A new constitutive equation for elastoviscoplastic fluid flows
}

Pierre Saramito

\section{To cite this version:}

Pierre Saramito. A new constitutive equation for elastoviscoplastic fluid flows. Journal of NonNewtonian Fluid Mechanics, 2007, 145 (1), pp.1-14. 10.1016/j.jnnfm.2007.04.004 • hal-00109101v4

\section{HAL Id: hal-00109101 https://hal.science/hal-00109101v4}

Submitted on 2 Apr 2007

HAL is a multi-disciplinary open access archive for the deposit and dissemination of scientific research documents, whether they are published or not. The documents may come from teaching and research institutions in France or abroad, or from public or private research centers.
L'archive ouverte pluridisciplinaire HAL, est destinée au dépôt et à la diffusion de documents scientifiques de niveau recherche, publiés ou non, émanant des établissements d'enseignement et de recherche français ou étrangers, des laboratoires publics ou privés. 


\title{
A new constitutive equation for elastoviscoplastic fluid flows
}

\author{
Pierre Saramito $^{\text {a }}$
}

${ }^{\mathrm{a}}$ CNRS - LJK, B.P. 53, 38041 Grenoble cedex 9, France

\begin{abstract}
From thermodynamic theory, a new three-dimensional model for elastoviscoplastic fluid flows is presented. It extends both the Bingham viscoplastic and the Oldroyd viscoelastic models. Fundamental flows are studied: simple shear flow, uniaxial elongation and large amplitude oscillatory shear. The complex moduli $\left(G^{\prime}, G^{\prime \prime}\right)$ are found to be in qualitative agreement with experimental data for materials that present microscopic network structures and large scale rearrangements. Various fluids of practical interest, such as liquid foams, droplet emulsions or blood, present such elastoviscoplastic behavior : at low stress, the material behaves as a viscoelastic solid, whereas at stresses above a yield stress, the material behaves as a fluid.
\end{abstract}

Keywords - non-Newtonian fluid; viscoelasticity; viscoplasticity; constitutive equation.

\section{Introduction}

\subsection{Historical background}

The development of viscoplastic rheological model based on yield stress started in 1900 when Schwedoff [20], studying a gelatin suspension, presented a one-dimensional plastic viscoelastic version of the Maxwell model:

$$
\left\{\begin{aligned}
\dot{\varepsilon} & =0, & & \text { when } \tau \leq \tau_{0}, \\
\lambda \frac{\mathrm{d} \tau}{\mathrm{d} t}+\left(\tau-\tau_{0}\right) & =\eta_{m} \dot{\varepsilon}, & & \text { when } \tau>\tau_{0},
\end{aligned}\right.
$$

where $\tau$ is the stress, $\dot{\varepsilon}$ the rate of deformation, $\eta_{m}>0$ the viscosity, $\tau_{0} \geq 0$ the yield stress and $\lambda \geq 0$ a relaxation time. In steady shear flow this reduces to $\tau=\tau_{0}+\eta_{m} \dot{\varepsilon}$ when $\tau>\tau_{0}$. In 1922, Bingham [1] proposed the one-dimensional stress-deformation rate equation for a viscous fluid with a yield stress:

$$
\max \left(0, \frac{|\tau|-\tau_{0}}{|\tau|}\right) \tau=\eta_{m} \dot{\varepsilon} \Longleftrightarrow \begin{cases}|\tau| \leq \tau_{0} & \text { when }|\dot{\varepsilon}|=0, \\ \tau=\eta_{m} \dot{\varepsilon}+\tau_{0} \frac{\dot{\varepsilon}}{|\dot{\varepsilon}|} & \text { otherwise. }\end{cases}
$$

Notice that this model is equivalent - up to the sign of $\dot{\varepsilon}$, assumed positive - to the steady case of the model proposed by Schwedoff. Numerous attempts have been made to modify this simple equation to

Email address: Pierre.Saramito@imag.fr (Pierre Saramito). 
account for more complex behavior of such materials. In 1926, Herschel and Bulkley [6] proposed to model the observed shear stress dependence of the viscosity on the shear rate $\dot{\varepsilon}$ after yielding by explicitly defining the viscosity $\eta_{m}$ as a power-law function of $|\dot{\varepsilon}|$.

In 1932, Prager [14], using the von Mises [24] yielding criterion, proposed to extend the Bingham model to the three-dimensional case and Oldroyd, in 1947, in a collection of papers (see e.g. [11]) studied the Bingham three-dimensional model and its Herschel-Bulkley extension coupled with the Navier-Stokes equations for the motion of the fluid. Oldroyd also proposed a three-dimensional constitutive equation which combines the yielding criterion together with a linear Hookean elastic behavior before yielding and a viscous behavior after yielding. In the one-dimensional case, the model can be written as:

$$
\left\{\begin{aligned}
\tau & =\mu \varepsilon \quad \text { when } \tau \leq \tau_{0}, \\
\left(\frac{|\tau|-\tau_{0}}{|\tau|}\right) \tau & =\eta_{m} \dot{\varepsilon} \quad \text { when } \tau>\tau_{0} .
\end{aligned}\right.
$$

When compared to (1), this model is an improvement, since the material is no longer rigid before yielding. Here, the yield stress $\tau_{0}$ is related to the critical strain $\varepsilon_{0}=\tau_{0} / \mu$. Since the first equation in (3) describes stresses in term of strain $\varepsilon$ and the second equation in (3) in term of strain rate $\dot{\varepsilon}$, the stress-strain curve predicted by this model must exhibit a discontinuity at the critical strain $\dot{\varepsilon}=\dot{\varepsilon}_{0}$ at which the stress jumps from $\tau=\tau_{0}$ to $\tau=\tau_{0}+\eta \dot{\varepsilon}$. This is an approximation of the true behavior of materials: the real deformation at the transition is expected to be smooth, at least continuous.

In 1950, Oldroyd [12] developed a theory for the invariant forms of rheological equations of state and proposed a three-dimensional viscoelastic model, that can be expressed in its one-dimensional version as:

$$
\lambda \frac{\mathrm{d} \tau}{\mathrm{d} t}+\tau=\eta_{m} \dot{\varepsilon}
$$

where the total stress $\sigma=\eta \dot{\varepsilon}+\tau$. In this approach the stress $\tau$ is the elastic part of the total stress, from which the elastic deformation can easily be obtained. The constant $\eta>0$ is another viscosity, often called the solvent viscosity in the context of polymer solutions.

\subsection{One-dimensional presentation of the proposed model}

Let us introduce an one-dimensional version of our model in order to combine the two previous models (2) and (4):

$$
\lambda \frac{\mathrm{d} \tau}{\mathrm{d} t}+\max \left(0, \frac{|\tau|-\tau_{0}}{|\tau|}\right) \tau=\eta_{m} \dot{\varepsilon} .
$$

where the total stress is $\sigma=\eta \dot{\varepsilon}+\tau$. When $\lambda=\eta=0$ we obtain (2) while when $\tau_{0}=0$ our model reduces to (4). Observe that (5) differs both from (1) and (3). Schwedoff proposed a rigid behavior $\dot{\varepsilon}=0$ when $|\tau| \leq \tau_{0}$ and Oldroyd proposed a brutal change of model when reaching the yield value. Our proposition assures a continuous change from a solid to a fluid behavior of the material.

The mechanical model is represented in Fig. 1.c. A friction $\tau_{0}$ has been inserted in the Oldroyd viscoelastic model (Fig. 1.b). At stresses below the yield stress, the friction element remains rigid. The level of the elastic strain energy required to break the friction element is determined by the von Mises yielding criterion. Consequently, before yielding, the whole system predicts only recoverable Kelvin-Voigt viscoelastic deformation due to the spring and the viscous element $\eta$ : the Kelvin-Voigt viscoelastic model is described by a spring and a viscous body in parallel (Fig. 1.a). The elastic behavior $\tau=\mu \varepsilon$ is expressed in (5) in differential form where $\mu=\eta_{m} / \lambda$ is the elasticity of the spring in Fig. 1.c. Before yielding, the total stress 
(a) Kelvin-Voigt

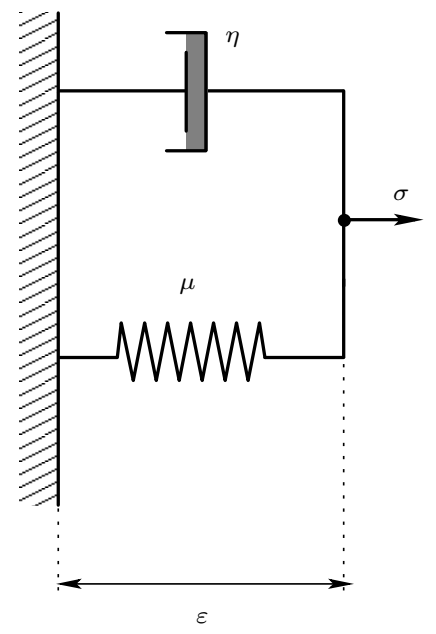

(c) present model

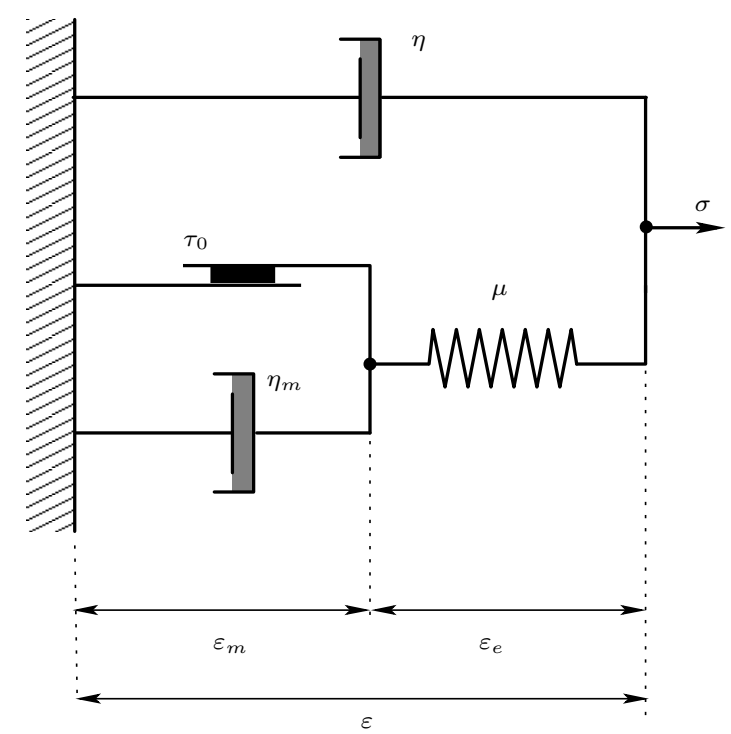

(b) Oldroyd

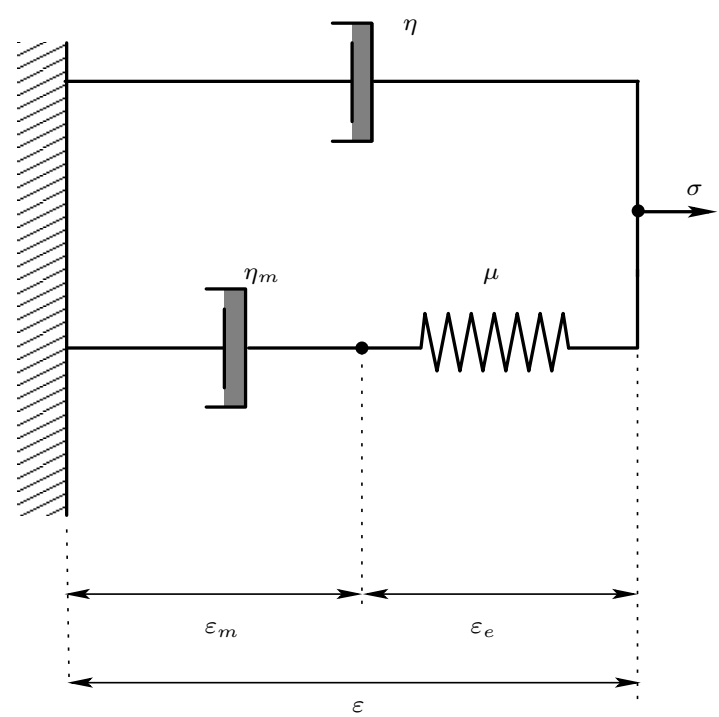

(d) creeping $\sigma(t)=\bar{\sigma}$ for the present model

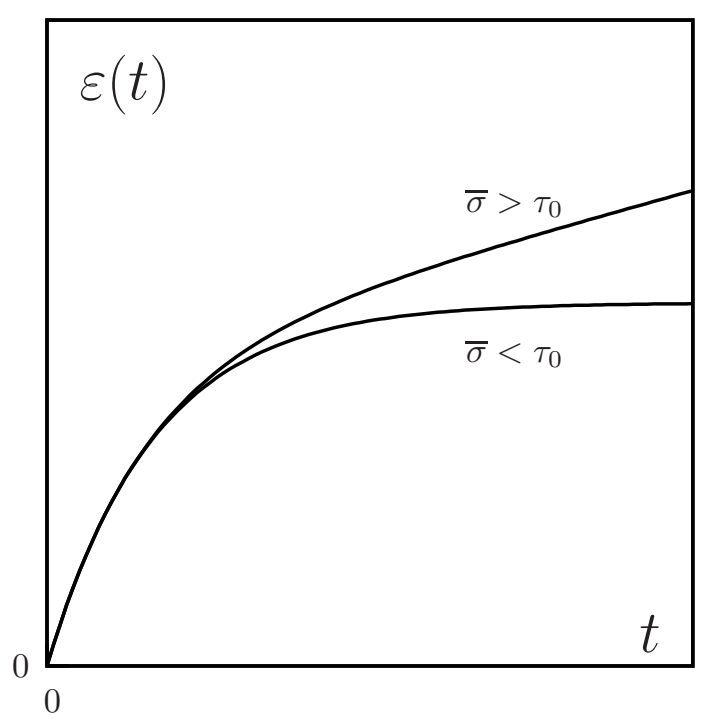

Figure 1. The proposed elastoviscoplastic model.

is $\sigma=\mu \varepsilon+\eta \dot{\varepsilon}+\tau$. As soon as the strain energy exceeds the level required by the von Mises criterion, the stress in the friction element attains the yield value and the element breaks allowing deformation of all the other elements. After yielding, the deformation of these elements describes the Oldroyd-type viscoelastic behavior. 
The evolution in time of elongation $\varepsilon(t)$ for a fixed imposed traction $\bar{\sigma}$ (creeping) is represented on Fig 1.d. When $\bar{\sigma} \leq \tau_{0}$, the elongation for a fixed imposed traction is bounded in time, which means that such material behaves as a solid. Otherwise, $\bar{\sigma}>\tau_{0}$, the elongation is unbounded in time, which means that the material behaves as a fluid. Notice the continuous behavior of the solution: the change of regime occurs at $t=t_{0}$ for the solution $\bar{\sigma}>\tau_{0}$ and there is neither jump nor loss of differentiability in the curve. The solution on Fig 1.d is computed explicitly and details are reported in appendix B.

\subsection{Comparison with other recent and closely related models}

A number of other closely related models have appeared in the literature.

In 1991, Beris et al.. [3], in order to recover a continuous approximation of the solution of the elasticviscoplastic model (3) proposed by Oldroyd, introduced an ad-hoc recovery procedure. Before yielding, the material behaves as an elastic solid while after yielding it behaves as a power-law viscous non-Newtonian fluid. Despite the lack of a thermodynamical analysis of their model, these authors have been able to propose, based on their computational results, a useful Cox-Merz rule extension that was found to be in good agreement with experimental data on a suspension of silicon particles in polyethylene.

In 2003, Puzrin and Houlsby [15, p. 254] inserted friction and a spring into the viscoelastic Kelvin-Voigt model and the resulting model is represented in Fig. 2.a. Before yielding, the friction is rigid and the material behaves as an elastic solid, thanks to the spring $\mu$. After yielding, the deformation is governed by the so-called standard model: a Kelvin-Voigt element plus a spring in series (see e.g. [10, p. 42]). The predicted elongation under a constant traction is presented on Fig. 2.b. The elongation jumps immediately at $t=0$ from $\varepsilon=0$ to $\varepsilon=\bar{\sigma} / \mu$, since the spring element has no time scale. Next, when $t>0$, if $\bar{\sigma} \leq \tau_{0}$, then the elongation remains constant, since the spring is fully extended. Otherwise, if $\bar{\sigma}>\tau_{0}$, the elongation grows and tends to a bounded value: the material behaves as a solid. By this way Puzrin and Houlsby were able to capture some relevant aspects of the behavior of saturated clays. These authors have proposed in a collection of papers $[7,16]$ many variants of their model. Nevertheless, it is not applicable to material such as human blood or liquid foams that deform under a low stress and flow under a sufficient stress.

In 1990 Isayev and Fan [9] proposed a viscoelastic plastic constitutive equation for flow of particles filled polymers. The main idea of these authors is to insert a friction and a spring into the viscoelastic Oldroyd model and the resulted model is represented on Fig. 2.c. Before yielding, the friction is rigid and the material behaves as a simple elastic solid, thanks to the spring $\mu$. After yielding, the deformation is described by the Oldroyd viscoelastic model. These authors have added in parallel other Maxwell elements in order to replace the Oldroyd viscoelastic element by a multi-mode Leonov viscoelastic model. The predicted elongation under a constant traction is presented on Fig. 2.d. As for the Puzrin-Houlsby model, the elongation jumps immediately at $t=0$ from $\varepsilon=0$ to $\varepsilon=\bar{\sigma} / \mu$. Next, when $t>0$, if $\bar{\sigma} \leq \tau_{0}$, then the elongation remains constant. Otherwise, if $\bar{\sigma}>\tau_{0}$, the elongation grows and is not bounded: in that case, the material behaves as a fluid. The friction element was able to describe the stress generated in the disperse phase of the filled polymer melts and this contribution has represented an important conceptual advance in this domain. Since the effect of added solid particles in a polymer melt is usually to reduce the viscoelasticity, the need of this kind of formulation arises primarily in the case of highly viscoelastic polymers such as rubbers. Nevertheless, the model presented in Fig. 1.c and. 1.d is a definite improvement: the instantaneous jump at $t=0$ followed by a constant elongation under traction of both the Puzrin-Houlsby and the one-mode simplified Isayev-Fan models is replaced by a smoother behavior.

During the last two decades, the thermodynamic framework developed and furnished some robust theoretical tools for managing efficiently rheological models: see e.g. [22, p. 26] or [10] for concise presentations 
(a) Puzrin-Houlsby

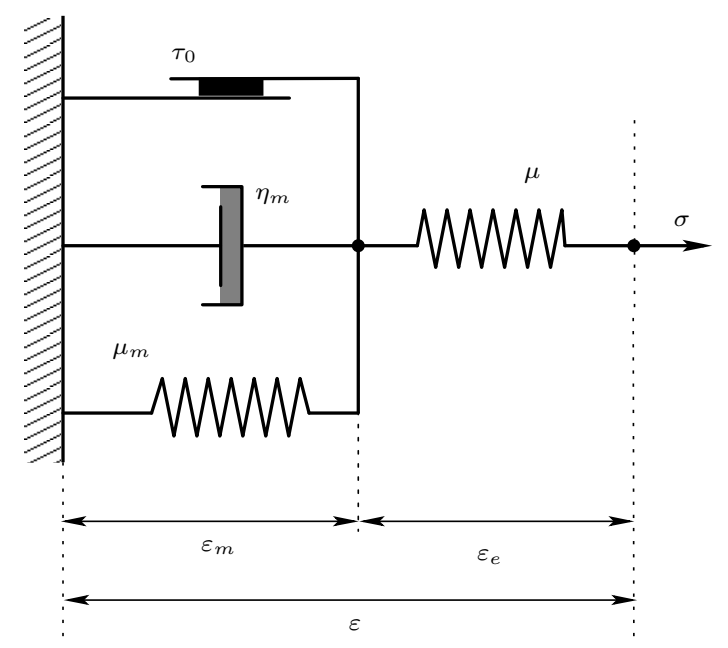

(c) Isayev-Fan (one Maxwell mode)

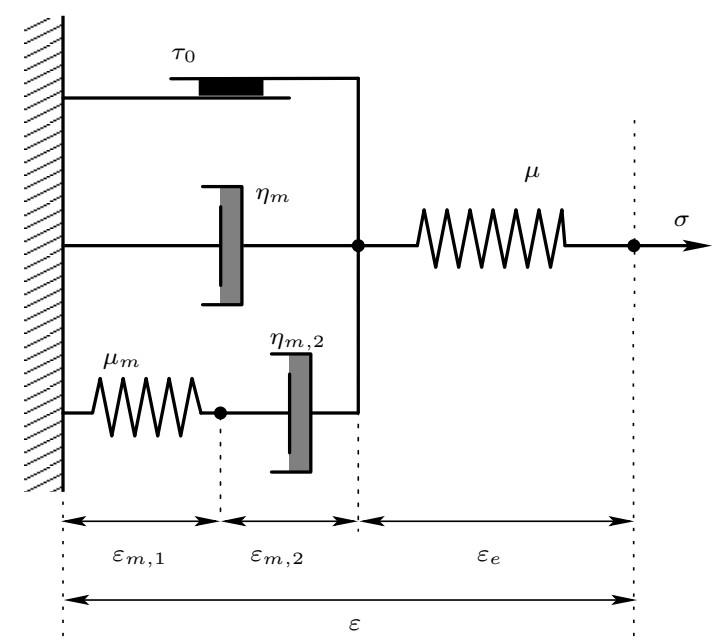

(b) Creeping for Puzrin-Houlsby model

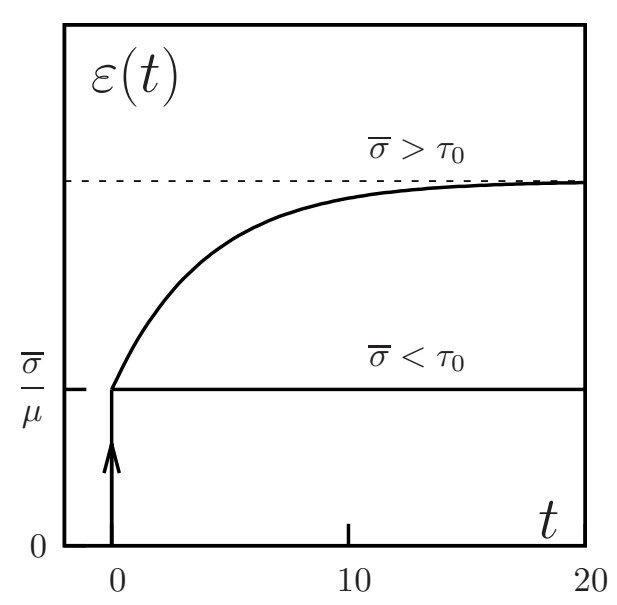

(d) Creeping for Isayev-Fan model

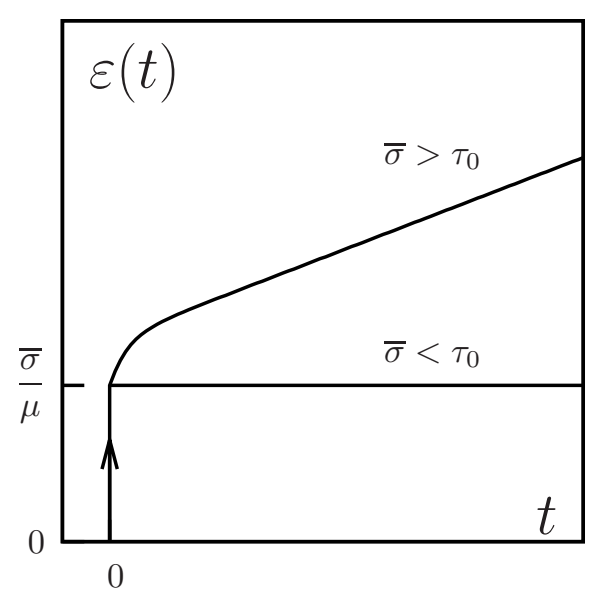

Figure 2. Some other elastoviscoplastic models.

of the thermodynamic framework for the development of combined viscoelastic and plastic models. The table 3 presents an historical summary of such models and signals their associated main features before and after yielding. The 3D column is marked when the model has been written in a general sense, e.g. with objective derivatives. The $\mathrm{TH}$ column is also marked when the model satisfies the second law of thermodynamics. The italic mark $X$ in the 3D or the TH collumns means that the corresponding property 


\begin{tabular}{|l|l|l|l|c|c|}
\hline year & contribution & before yielding & after yielding & 3D & TH \\
\hline \hline 1900 & Schwedoff [20] & rigid solid & Maxwell viscoelastic fluid & & \\
\hline 1922 & Bingham [1] & rigid solid & Newtonian fluid & $X$ & $X$ \\
\hline 1926 & Herschel-Bulkley [6] & rigid solid & power-law fluid & $X$ & $X$ \\
\hline 1947 & Oldroyd [11] & elastic solid & Newtonian fluid & X & X \\
\hline 1990 & Isayev-Fan [9] & elastic solid & Leonov viscoelastic fluid & X & X \\
\hline 1991 & Beris et al. [3] & elastic solid & power-law fluid & X & \\
\hline 2003 & Puzrin-Houlsby [15, p. 254] & elastic solid & Kelvin viscoelastic solid & $\mathbf{X}$ & $\mathbf{X}$ \\
\hline 2007 & present & Kelvin-Voigt viscoelastic solid & Oldroyd viscoelastic fluid & $\mathbf{X}$ & $\mathbf{X}$ \\
\hline
\end{tabular}

Figure 3. Summary table of the referenced contributions on elastoviscoplastic models.

has been showed later. For instance, the Bingham and the Herschel-Bulkley models was first proposed in a one-dimensional context, next extended to 3D and finally founded to satisfy the second law of thermodynamics.

The aim of the present article is to build the proposed model for the general three-dimensional case (section 2) and to study it on three simple flows (section 3): a simple shear flow, an uniaxial elongation and an oscillatory shear flow. The impatient reader - and the reader who is unfamiliar with the thermodynamic framework - could jump directly to the end of section 2 where the complete set of equations (11) governing such a flow is presented, before reading section 3 devoted to applications.

There are also two technical appendices. Appendix A contains some properties used in section 2 and related to multi-dimensional subdifferential calculus. Appendix B groups the explicit resolution of the one-dimensional creeping problems as presented in the introduction on Figs. 1 and 2.

\section{The proposed model}

\subsection{Thermodynamic framework}

The state of the system is described by using two independent variables : the total deformation tensor $\varepsilon$ and an internal variable, the elastic deformation tensor $\varepsilon_{e}$. We have $\varepsilon=\varepsilon_{e}+\varepsilon_{m}$ where $\varepsilon_{m}$ represents the plastic deformation tensor. Following Halphen and Nguyen [5] (see e.g. [22] or [10, p. 97]) we say that a generalized standard material is characterized by the existence of a free energy function $\mathcal{E}$ and a potential of dissipation $\mathcal{D}$, that are both convex functions of their arguments. The proposed model that combines viscoelasticity and viscoplasticity, is represented by a rheological scheme on Fig. 1.c and can be written as:

$$
\begin{aligned}
& \mathcal{E}\left(\varepsilon, \varepsilon_{e}\right)=\mu\left|\varepsilon_{e}\right|^{2}, \\
& \mathcal{D}\left(\dot{\varepsilon}, \dot{\varepsilon}_{e}\right)=\varphi(\dot{\varepsilon})+\varphi_{m}\left(\dot{\varepsilon}-\dot{\varepsilon}_{e}\right),
\end{aligned}
$$

where $\mu>0$ is the elasticity parameter and where $|$.$| denotes the matrix norm, defined by a double$ contraction of indices : $\left|\varepsilon_{e}\right|^{2}=\varepsilon_{e}: \varepsilon_{e}$. The functions $\varphi$ and $\varphi_{m}$ are expressed by : 


$$
\varphi(\dot{\varepsilon})=\left\{\begin{array}{ll}
\eta|\dot{\varepsilon}|^{2} & \text { when } \operatorname{tr} \dot{\varepsilon}=0, \\
+\infty & \text { otherwise, }
\end{array} \quad \text { and } \quad \varphi_{m}\left(\dot{\varepsilon}_{m}\right)= \begin{cases}\eta_{m}\left|\dot{\varepsilon}_{m}\right|^{2}+\tau_{0}\left|\dot{\varepsilon}_{m}\right| & \text { when } \operatorname{tr} \dot{\varepsilon}_{m}=0, \\
+\infty & \text { otherwise }\end{cases}\right.
$$

The $\varphi$ function expresses the incompressible viscous behavior at macroscopic level and is associated to a macroscopic viscosity $\eta>0$ while the $\varphi_{m}$ function expresses the viscoplastic behavior at microscopic level, by using a microscopic viscosity $\eta_{m}>0$, acting on continuous modification of the network links. and also a yield stress value $\tau_{0} \geq 0$. When the stress becomes higher than this value, some topological modifications appear in the network of contacts. This model satisfies the second law of thermodynamics: in the framework of generalized standard materials $[5,22,10]$ this property is a direct consequence of the convexity of both $\mathcal{E}$ and $\mathcal{D}$.

\subsection{The constitutive law}

Let $\Omega$ be a bounded domain of $\mathbb{R}^{N}$, where $N=1,2,3$. Since both $\varphi$ and $\varphi_{m}$ are non-linear and nondifferentiable, the following manipulations involve subdifferential calculus from convex analysis. The material constitutive laws can be written as:

$$
\sigma \in \frac{\partial \mathcal{E}}{\partial \varepsilon}+\frac{\partial \mathcal{D}}{\partial \dot{\varepsilon}} \quad \text { and } \quad 0 \in \frac{\partial \mathcal{E}}{\partial \varepsilon_{e}}+\frac{\partial \mathcal{D}}{\partial \dot{\varepsilon}_{e}},
$$

where $\sigma$ is the total Cauchy stress tensor. Using definition (6) of $\mathcal{E}$ and $\mathcal{D}$, we get:

$$
\sigma \in \partial \varphi(\dot{\varepsilon})+\partial \varphi_{m}\left(\dot{\varepsilon}-\dot{\varepsilon}_{e}\right) \quad \text { and } \quad 0 \in 2 \mu \varepsilon_{e}-\partial \varphi_{m}\left(\dot{\varepsilon}-\dot{\varepsilon}_{e}\right) .
$$

The combination of the two previous relations leads to $\sigma-2 \mu \varepsilon_{e} \in \partial \varphi(\dot{\varepsilon})$. Then, by using expression (A.3) of $\partial \varphi$ from the technical annex, and by introducing the pressure field $p$, we get the following expression of the total Cauchy stress tensor: $\sigma=-p \cdot I+2 \eta \dot{\varepsilon}+2 \mu \varepsilon_{e}$ when $\operatorname{tr}(\dot{\varepsilon})=0$. Then, the second relation in (9) is equivalent to $\dot{\varepsilon}-\dot{\varepsilon}_{e} \in \partial \varphi_{m}^{*}\left(2 \mu \varepsilon_{e}\right)$ where $\varphi_{m}^{*}$ is the dual of $\varphi_{m}$. Let us introduce the elastic stress tensor $\tau=2 \mu \varepsilon_{e}$. The expression (A.2) of $\partial \varphi_{m}^{*}$ in annex yields:

$$
\lambda \dot{\tau}+\max \left(0, \frac{\left|\tau_{d}\right|-\tau_{0}}{\left|\tau_{d}\right|}\right) \tau=2 \eta_{m} \dot{\varepsilon}
$$

where $\lambda=\eta_{m} / \mu$ is the relaxation time and $\tau_{d}=\tau-\frac{1}{N} \operatorname{tr}(\tau) I$ denotes the deviatoric part of $\tau$.

\subsection{The system of equations}

Since the material is considered in large deformations, we choose to use the Eulerian mathematical framework, more suitable for fluids flows computations. We assume that $\dot{\varepsilon}=D(\mathbf{v})=\left(\nabla \mathbf{v}+\nabla \mathbf{v}^{T}\right) / 2$ is the rate of deformation, while the material derivative $\dot{\tau}$ of tensor $\tau$ in the Eulerian framework is expressed by the Gordon-Schowalter derivative [4] $: \stackrel{\square}{\tau}=\frac{\partial \tau}{\partial t}+\mathbf{v} \cdot \nabla \tau+\tau W(\mathbf{v})-W(\mathbf{v}) \tau-a(\tau D(\mathbf{v})+D(\mathbf{v}) \tau)$ where $W(\mathbf{v})=\left(\nabla \mathbf{v}-\nabla \mathbf{v}^{T}\right) / 2$ is the vorticity tensor. The material parameter $a \in[-1,1]$ is associated to the Gordon-Schowalter's derivative. When $a=0$ we obtain the Jaumann derivative of tensors, while $a=1$ and $a=-1$ are associated to the upper and the lower convected derivatives, respectively.

The elastoviscoplastic fluid is then described by a set of three equations associated to three unknowns $(\tau, \mathbf{v}, p)$ : the differential equation (10) is completed with the conservation of momentum and mass: 


$$
\left\{\begin{aligned}
\lambda \stackrel{\square}{\tau}+\max \left(0, \frac{\left|\tau_{d}\right|-\tau_{0}}{\left|\tau_{d}\right|}\right) \tau-2 \eta_{m} D(\mathbf{v}) & =0, \\
\rho\left(\frac{\partial \mathbf{v}}{\partial t}+\mathbf{v} \cdot \nabla \mathbf{v}\right)-\operatorname{div}(-p I+2 \eta D(\mathbf{v})+\tau) & =\mathbf{f}, \\
\operatorname{div} \mathbf{v} & =0,
\end{aligned}\right.
$$

where $\rho$ denotes the constant density and $\mathbf{f}$ a known external force, such as the gravity. These equations are completed by some suitable initial and boundaries conditions in order to close the system. For instance the initial conditions $\tau(t=0)=\tau_{0}$ and $\mathbf{v}(t=0)=\mathbf{v}_{0}$ and the boundary condition $\mathbf{v}=\mathbf{v}_{\Gamma}$ on the boundary $\partial \Omega$ are convenient. The total Cauchy stress tensor can be written as:

$$
\sigma=-p I+2 \eta D(\mathbf{v})+\tau .
$$

Notice that when $\tau_{0}=0$ the model reduces to the usual viscoelastic Oldroyd model $[12,18]$ and when $\lambda=0$ it reduces to the viscoplastic Bingham model $[1,17]$. When both $\tau_{0}=0$ and $\lambda=0$ the fluid is Newtonian and the set of equations reduces to the classical Navier-Stokes equations with a total viscosity $\eta_{0}=\eta+\eta_{m}$. Conversely, when both $\tau_{0} \neq 0$ and $\lambda \neq 0$ the fluid is elastoviscoplastic.

Dimensionless formulation - Let us introduce some classical dimensionless numbers:

$$
W e=\frac{\lambda U}{L}, B i=\frac{\tau_{0} L}{\eta_{0} U} \text { and } R e=\frac{\rho U L}{\eta_{0}},
$$

where $U$ and $L$ are some characteristic velocity and length of the flow, respectively. We also use the retardation parameter $\alpha=\eta_{m} / \eta_{0}$. The problem reduces to that of finding dimensionless fields, also denoted by $(\tau, \mathbf{v}, p)$ such that:

$$
\left\{\begin{aligned}
W e \stackrel{\square}{\tau}+\max \left(0, \frac{\left|\tau_{d}\right|-B i}{\left|\tau_{d}\right|}\right) \tau-2 \alpha D(\mathbf{v}) & =0, \\
\operatorname{Re}\left(\frac{\partial \mathbf{v}}{\partial t}+\mathbf{v} \cdot \nabla \mathbf{v}\right)-\operatorname{div}(-p I+2(1-\alpha) D(\mathbf{v})+\tau) & =\mathbf{f}, \\
\operatorname{div} \mathbf{v} & =0,
\end{aligned}\right.
$$

where $\mathbf{f}$ denotes some known dimensionless vector field. These equations are completed by the initial and boundaries conditions.

\section{Examples}

\subsection{Uniaxial elongation}

The fluid is at the rest at $t=0$ and a constant elongational rate $\dot{\varepsilon}_{0}$ is applied: the Weissenberg number is $W e=\lambda \dot{\varepsilon}_{0}$ and the Bingham number $B i=\tau_{0} /\left(\eta_{0} \dot{\varepsilon}_{0}\right)$. All quantities presented in this paragraph are dimensionless.

The flow is three-dimensional and the dimensionless velocity gradient writes $\nabla \mathbf{v}=\operatorname{diag}(1,-1 / 2,-1 / 2)$. The problem reduces to find $\tau_{11}, \tau_{22}$ and $\tau_{33}$ such that

$$
\left\{\begin{array}{l}
W e \frac{\mathrm{d} \tau_{11}}{\mathrm{~d} t}+(\kappa-2 a W e) \tau_{11}=2 \alpha, \\
W e \frac{\mathrm{d} \tau_{k k}}{\mathrm{~d} t}+(\kappa+a W e) \tau_{k k}=-\alpha, \quad k=2,3
\end{array}\right.
$$



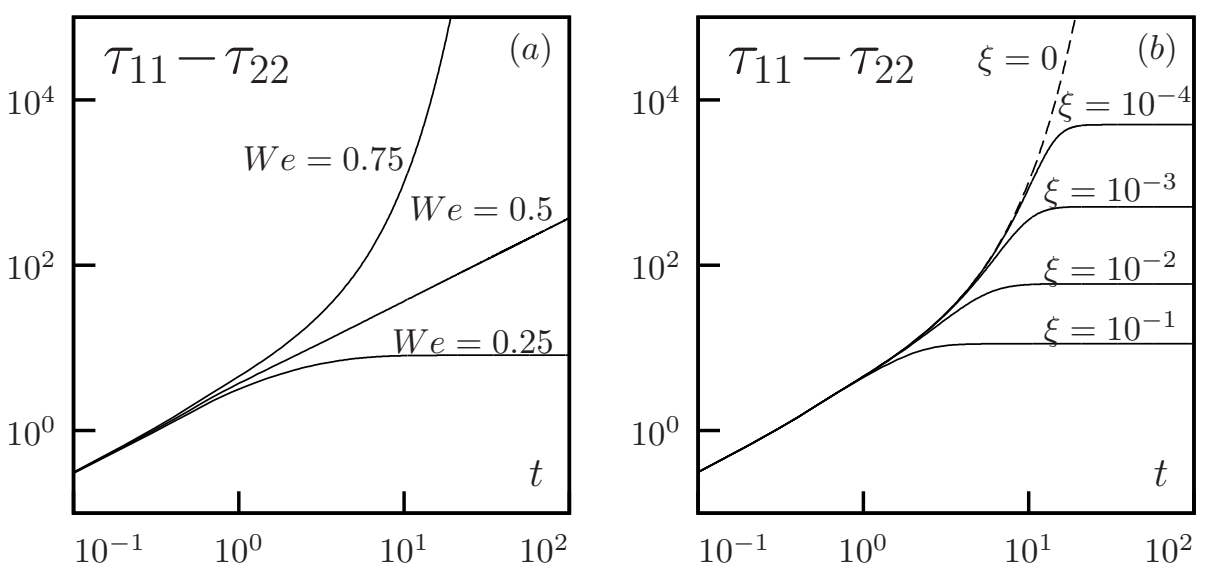

Figure 4. Dimensionless first normal stress difference $\psi=\tau_{11}-\tau_{22}$ for uniaxial elongation when $B i=1$, $a=1$ and $\alpha=1$ : (a) influence of $W e$ for $\xi=0$; (b) influence of $\xi$ for $W e=0.75$.

with the initial condition $\tau(t=0)=0$ and where $\kappa=\max \left(0,1-B i /\left|\tau_{d}\right|\right)$. As above, since $\tau(0)=0$ and $\tau(t)$ is continuous, there exists $t_{0}>0$ such that when $t \in\left[0, t_{0}\right]$ we have $\left|\tau_{d}\right| \leq B i$ and thus $\kappa=0$ : this is the linear flow regime. The eigenvalues of the system are $-2 a W e$ and $a W e$. For $t>t_{0}$, the case $\kappa>0$ occurs. Since $\kappa \leq 1$, when $a W e>1 / 2$ the stress becomes unbounded in finite time, as shown on Fig. 4.a that plots the dimensionless first normal stress difference $\psi=\tau_{11}-\tau_{22}$. This drawback is still true when $B i=0$, i.e. for the Oldroyd viscoelastic model. In the context of viscoelastic models, some alternate constitutive equations that extend the Oldroyd model have been proposed. Let us consider the following constitutive equation:

$$
W e \stackrel{\square}{\tau}+(1+\xi \operatorname{tr} \tau) \max \left(0, \frac{\left|\tau_{d}\right|-B i}{\left|\tau_{d}\right|}\right) \tau-2 \alpha D(\mathbf{v})=0,
$$

where $\xi \geq 0$ is a new material parameter. For $B i=0$ we obtain the viscoelastic Phan-Thien and Tanner model (see e.g. $[13,19]$ ). For $\xi=0$ we obtain the previous elastoviscoplastic model (11) while for any $\xi>0$ the solution remains bounded, as shown on Fig. 4.b for $W e=0.75$.

\subsection{Simple shear flow}

The fluid is at the rest at $t=0$ and a constant shear rate $\dot{\gamma}_{0}$ is applied: the Weissenberg number is $W e=\lambda \dot{\gamma}_{0}$ and the Bingham number $B i=\tau_{0} /\left(\eta_{0} \dot{\gamma}_{0}\right)$.

The flow is two-dimensional and the dimensionless velocity gradient is constant: $\nabla \mathbf{v}=([0,1] ;[0,0])$. The problem reduces to finding $\tau_{11}, \tau_{22}$ and $\tau_{12}$, such that, for all $t>0$ :

$$
W e \frac{\mathrm{d}}{\mathrm{d} t}\left(\begin{array}{c}
\tau_{11} \\
\tau_{22} \\
\tau_{12}
\end{array}\right)+W e\left(\begin{array}{ccc}
0 & 0 & -(1+a) \\
0 & 0 & 1-a \\
\frac{1-a}{2} & -\frac{1+a}{2} & 0
\end{array}\right)\left(\begin{array}{c}
\tau_{11} \\
\tau_{22} \\
\tau_{12}
\end{array}\right)+\kappa\left(\begin{array}{c}
\tau_{11} \\
\tau_{22} \\
\tau_{12}
\end{array}\right)=\left(\begin{array}{c}
0 \\
0 \\
\alpha
\end{array}\right)
$$



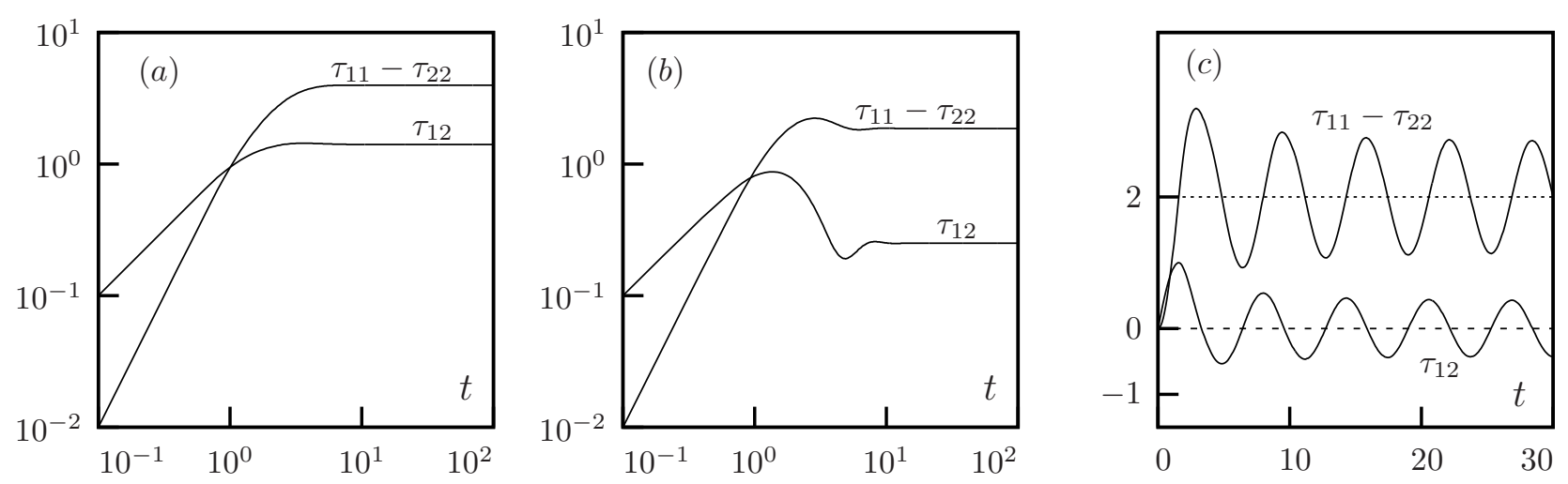

Figure 5. Simple shear flow for $W e=1$ and $\alpha=8 / 9$ : (a) $a=1$ and $B i=1$; (b) $a=0$ and $B i=1 ;(\mathrm{c}) a=0$ and $B i=2$.

with the initial condition $\tau(0)=0$ and where $\kappa=\max \left(0,1-B i /\left|\tau_{d}\right|\right)$ and $\left|\tau_{d}\right|^{2}=(1 / 2)\left(\tau_{11}-\tau_{22}\right)^{2}+2 \tau_{12}^{2}$. Let $\psi=\tau_{11}-\tau_{22}$ be the dimensionless normal stress difference. Then $\tau_{11}=\frac{1+a}{2} \psi$ and $\tau_{22}=-\frac{1-a}{2} \psi$. The solution $\left(\tau_{12}, \psi\right)$ is represented on Fig. 5. Since $\tau(0)=0$ and $\tau(t)$ is continuous, there exists $t_{0}>0$ such that when $t \in\left[0, t_{0}\right]$ we have $\left|\tau_{d}\right| \leq B i$ and thus $\kappa=0$ : this is the linear flow regime. The eigenvalues of the system are 0 and $\pm i \sqrt{1-a^{2}}$. At $t=t_{0},\left|\tau_{d}\right|$ reaches $B i$. Then, for $t>t_{0}$, the non-linear factor $\kappa>0$ occurs: the corresponding term reduces the growth of the solution, which now remains bounded. When $a=1$ (see Fig. 5.a, where $\tau_{22}=0$ ) and when $a=0$ and $B i$ (see Fig. 5.b, where $\tau_{22}=-\tau_{11}$ ) is small enough, the solution tends to a constant. Remark the important overshoot of both $\tau_{12}$ and $\tau_{11}-\tau_{22}$ when $a=0$ (Fig. 5.b) while when $a=1$ there is only a tiny overshoot of $\tau_{12}$. When $a=0$ and $B i$ becomes large, instabilities appear, while the solution remains bounded (Fig. 5.c). There has been many studies of the $a$ parameter of the Gordon-Schowalter derivative (see e.g. [23]). Based on comparisons with data measurements, most authors consider that only the case $a=1$ - or at least $a$ close to 1 - is relevant from a practical point of view.

Notice that the dimensionless steady shear viscosity $\eta_{s} / \eta_{0}$ coincides with the dimensionless shear stress $\sigma_{12}=1-\alpha+\tau_{12}$. When the solution becomes stationary, let us observe the dimensionless steady shear viscosity $\eta_{s} / \eta_{0}$ as a function of $W e$ on Fig. 6 . The material presents a shear thinning character. For large $W e$, the shear viscosity tends to a plateau when $\xi=0$ (Fig. 6.a) and decreases monotonically when $\xi>0$ (Fig. 6.b). This shear thinning behavior is more pronounced when $B i$ increases: the value of $B i$ controls the plateau at small values of $W e$.

\subsection{Periodic shear flow}

An oscillatory shear flow is applied: the imposed shear strain is $\gamma_{0} \sin (\omega t)$ and the shear rate becomes $\gamma_{0} \omega \cos (\omega t)$. The Weissenberg number is defined by $W e=\lambda \omega$ and the Bingham number by $B i=$ $\tau_{0} /\left(\eta_{0} \gamma_{0} \omega\right)$.

The flow is two-dimensional and the velocity gradient is periodic: $\nabla \mathbf{v}(t)=([0, \cos t] ;[0,0])$. The problem reduces to that of finding $\tau_{11}, \tau_{22}$ and $\tau_{12}$, from $\mathbb{R}^{+}$to $\mathbb{R}$, such that 

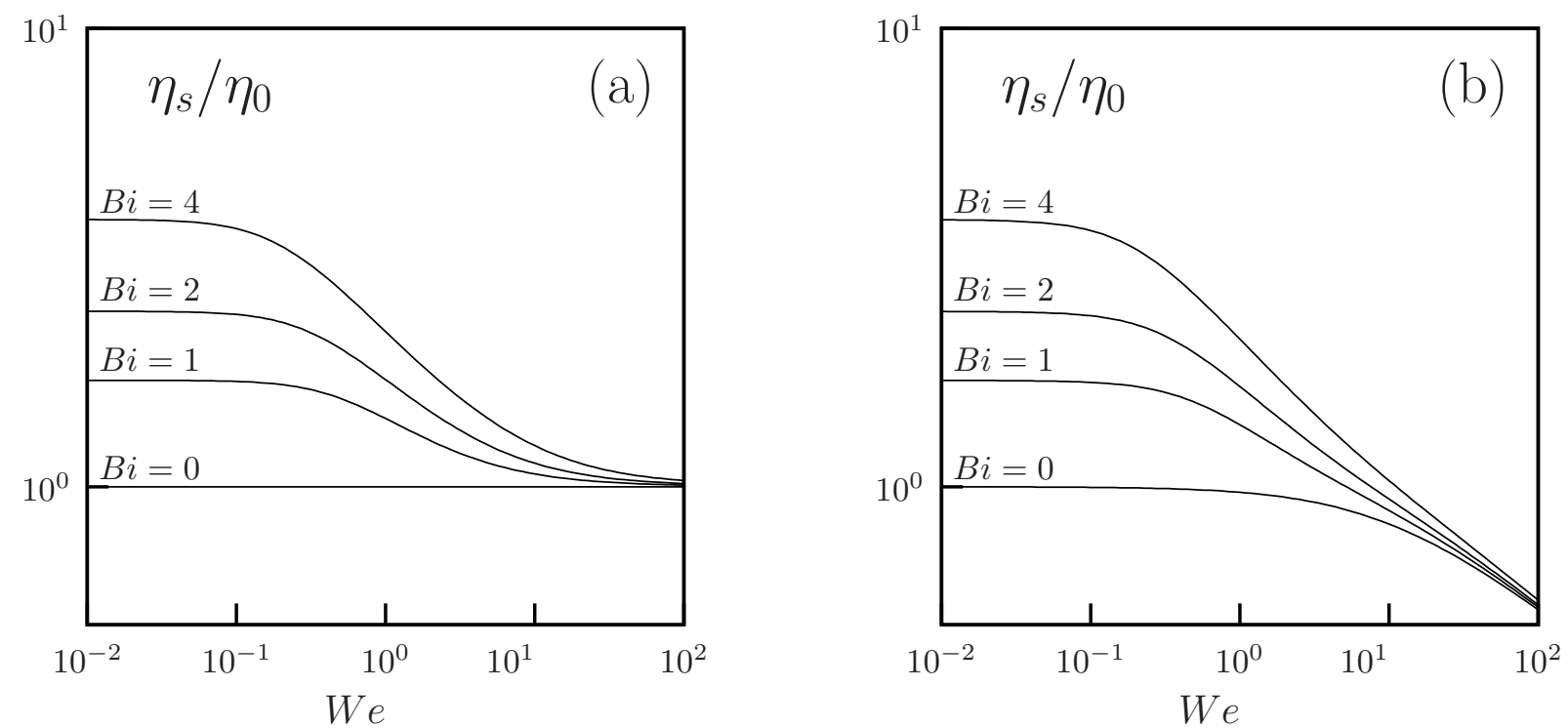

Figure 6. Shear viscosity for $a=1, \alpha=1$ and (a) $\xi=0$; (b) $\xi=0.015$.

$$
\left\{\begin{aligned}
W e \frac{\mathrm{d}}{\mathrm{d} t}\left(\begin{array}{c}
\tau_{11} \\
\tau_{22} \\
\tau_{12}
\end{array}\right)+\mathcal{F}(t, \tau) & \left.=\left(\begin{array}{c}
0 \\
0 \\
\alpha \cos t
\end{array}\right) \quad \text { in }\right] 0,2 \pi[, \\
\tau(0) & =\tau(2 \pi),
\end{aligned}\right.
$$

where

$$
\mathcal{F}(t, \tau)=W e \cos (t) \mathcal{A}_{a}\left(\begin{array}{c}
\tau_{11} \\
\tau_{22} \\
\tau_{12}
\end{array}\right)+\kappa\left(\begin{array}{c}
\tau_{11} \\
\tau_{22} \\
\tau_{12}
\end{array}\right)
$$

and $\kappa=\max \left(0,1-B i /\left|\tau_{d}\right|\right)$ and $\left|\tau_{d}\right|^{2}=(1 / 2)\left(\tau_{11}-\tau_{22}\right)^{2}+2 \tau_{12}^{2}$. The $\xi$ material parameter introduced in (12) has been found to have an influence on the elongational flow, while in the case of the periodic shear flow, it has been found to have very minor influence. Thus only the case $\xi=0$ is presented in this section.

\subsubsection{The solution for $B i=0$}

The case $B i=0$ is asymptotically reached when the amplitude $\gamma_{0}$ becomes large. The material behaves as a viscoelastic fluid and the system reduces to:

$$
\left\{\begin{aligned}
W e \frac{\mathrm{d} \tau_{11}}{\mathrm{~d} t}-W e(1+a) \cos (t) \tau_{12}+\tau_{11} & =0, \\
W e \frac{\mathrm{d} \tau_{22}}{\mathrm{~d} t}+W e(1-a) \cos (t) \tau_{12}+\tau_{22} & =0, \\
W e \frac{\mathrm{d} \tau_{12}}{\mathrm{~d} t}+\frac{W e \cos (t)}{2}\left((1-a) \tau_{11}-(1+a) \tau_{22}\right)+\tau_{12} & =\alpha \cos (t),
\end{aligned}\right.
$$


together with periodic boundary condition $\tau(0)=\tau(2 \pi)$. By introducing the first normal stress difference $\psi=\tau_{11}-\tau_{22}$, the system can be rewritten in a more compact form:

$$
\left\{\begin{array}{c}
W e \frac{\mathrm{d} \psi}{\mathrm{d} t}+\psi=2 W e \cos (t) \tau_{12} \\
W e \frac{\mathrm{d} \tau_{12}}{\mathrm{~d} t}+\tau_{12}=\alpha \cos (t)-\frac{W e\left(1-a^{2}\right) \cos (t)}{2} \psi \\
\psi(0)=\psi(2 \pi) \text { and } \tau_{12}(0)=\tau_{12}(2 \pi) \\
\tau_{11}=\frac{1+a}{2} \psi \text { and } \tau_{22}=-\frac{1-a}{2} \psi
\end{array}\right.
$$

When $a= \pm 1$ the system admits an explicit solution:

$$
\begin{aligned}
\psi(t) & =\frac{\alpha W e}{\left(1+W e^{2}\right)\left(1+4 W e^{2}\right)}\left(\left(1+2 W e^{2}\right) \cos (2 t)+3 W e \sin (2 t)+1+4 W e^{2}\right), \\
\tau_{12}(t) & =\frac{\alpha}{1+W e^{2}}(\cos (t)+W e \sin (t)) .
\end{aligned}
$$

Otherwise, when $|a|<1$, the solution has no explicit form to our knowledge.

\subsubsection{The solution for large $B i$}

The case $B i \rightarrow+\infty$ is reached when the amplitude $\gamma_{0}$ becomes small. The material behaves as an elastic solid. Moreover, there exists a finite value $B i_{c}>0$ such that when $B i>B i_{c}$ then the solution satisfies $\left|\tau_{d}\right|<B i$. In that case, the system reduces to:

$$
\left\{\begin{aligned}
W e \frac{\mathrm{d} \tau_{11}}{\mathrm{~d} t}-W e(1+a) \cos (t) \tau_{12} & =0, \\
W e \frac{\mathrm{d} \tau_{22}}{\mathrm{~d} t}+W e(1-a) \cos (t) \tau_{12} & =0, \\
W e \frac{\mathrm{d} \tau_{12}}{\mathrm{~d} t}+\frac{W e \cos (t)}{2}\left((1-a) \tau_{11}-(1+a) \tau_{22}\right) & =\alpha \cos (t),
\end{aligned}\right.
$$

together with the periodic boundary condition $\tau(0)=\tau(2 \pi)$. Let $\psi=\tau_{11}-\tau_{22}$. Combining the previous equations, we get

$$
\left\{\begin{array}{c}
W e \frac{\mathrm{d} \psi}{\mathrm{d} t}=2 W e \cos (t) \tau_{12}, \\
W e \frac{\mathrm{d} \tau_{12}}{\mathrm{~d} t}=\alpha \cos (t)-\frac{W e\left(1-a^{2}\right) \cos (t)}{2} \psi \\
\psi(0)=\psi(2 \pi) \text { and } \tau_{12}(0)=\tau_{12}(2 \pi) \\
\tau_{11}=\frac{1+a}{2} \psi \text { and } \tau_{22}=-\frac{1-a}{2} \psi
\end{array}\right.
$$

When $a= \pm 1$ the solution is known explicitly:

$$
\begin{aligned}
\psi(t) & =\frac{\alpha(c-\cos (2 t))}{2 W e}, \\
\tau_{12}(t) & =\frac{\alpha \sin (t)}{W e},
\end{aligned}
$$

where $c$ is an arbitrary constant. In that case $\left|\tau_{d}(t)\right|$ is maximum for $t=\pi / 2$ when $c \geq 0$ and $t=-\pi / 2$ when $c \leq 0$. We have:

$$
\max _{t \in[0,2 \pi]}\left|\tau_{d}(t)\right|=\frac{\alpha}{2 \sqrt{2} W e}\left((1+|c|)^{2}+16\right)^{1 / 2} .
$$


Let

$$
B i_{c}=\sqrt{\frac{17}{8}} \frac{\alpha}{W e} .
$$

Then, when $B i \geq B i_{c}$, there exists a periodic solution $\tau(t)$ for all $t$ in $[0,2 \pi]$ such that $\left|\tau_{d}(t)\right| \leq B i$ in $[0,2 \pi]$ and this solution is expressed by (17)-(18).

\subsubsection{The solution for $B i \in] 0, B i_{c}[$}

Problem (14) is approximated by using a centered second order scheme:

$$
\left\{\begin{array}{c}
\frac{W e}{2 \Delta t}\left(\begin{array}{c}
\tau_{11}^{n+1}-\tau_{11}^{n-1} \\
\tau_{22}^{n+1}-\tau_{22}^{n-1} \\
\tau_{12}^{n+1}-\tau_{12}^{n-1}
\end{array}\right)+\mathcal{F}\left(t_{n}, \tau^{n}\right)=\left(\begin{array}{c}
0 \\
0 \\
\alpha \cos t_{n}
\end{array}\right), \\
\tau_{0}=\tau_{N},
\end{array}\right.
$$

with $t_{n}=2 \pi n / N, n=0 \ldots N, \Delta t=2 \pi / N$ and $\tau^{n} \approx \tau\left(t_{n}\right)$. This is a $N$ by $N$ non-linear system of equations that we solve by using a non-linear Gauss-Seidel iterative algorithm. We use $N=1000$ points in the $[0,2 \pi]$ interval, and iterations are stopped when the maximal residual term in the discrete nonlinear problem (19) becomes lower than $10^{-12}$. Fig. 7 shows the solution $\tau_{12}$ and $\tau_{11}$ (while $\tau_{22}=0$ ) for $a=1, B i=3, \alpha=1 \xi=0$, and $W e=0.1,0.05$ and 0.025 . When $a=0$ the components $\tau_{12}$ and $\tau_{11}$ are comparable up to a slight variation, while $\tau_{22}=-\tau_{11}$, and the corresponding solution is not represented.

\subsubsection{Fourier analysis}

We expand the solution $\sigma_{12}=(1-\alpha) \dot{\gamma}+\tau_{12}$ as:

$$
\sigma_{12}(t)=\sum_{k \geq 1} a_{k} \sin (k t)+b_{k} \cos (k t)
$$

where the coefficients are expressed by:

$$
a_{k}=\frac{1}{\pi} \int_{0}^{2 \pi} \sigma_{12}(t) \sin (k t) \mathrm{d} t \quad \text { and } \quad b_{k}=\frac{1}{\pi} \int_{0}^{2 \pi} \sigma_{12}(t) \cos (k t) \mathrm{d} t .
$$

When the response is linear we have $a_{k}=b_{k}=0$ for all $k \geq 2$. There are two cases when this situation occurs: when $B i=0$ while the material behaves as a viscoelastic fluid, and when $B i \rightarrow+\infty$ while its behaves as an elastic solid. Otherwise, only odd harmonics appear. In [8], the authors propose large amplitude oscillatory shear as a way to classify complex fluids. Following this approach, we observe on Fig. 9 that the third harmonic contributes of about $20 \%$ of the first harmonic, and higher harmonics decay very fast. See also [25, p. 74] for a comparable observation, based on a comparison between experimental data on a linear hight-density polyethylene melt and several theoretical models. See also Fig. 11 in [8] for a similar observation based on experimental data for a Xanthan gum solution. Therefore, the moduli obtained from the first harmonic via Fourier transformation analysis are not substantially different from the moduli calculated neglecting higher harmonics, as in the linear regime. By extension to the linear regimes, let us introduce the in-phase moduli $G^{\prime}$ and the out-of-phase moduli $G^{\prime \prime}$ : these are related to the first Fourier coefficients by $G^{\prime}=a_{1}$ and $G^{\prime \prime}=b_{1}$. When $B i \geq B i_{c}$, the material behaves as a viscoelastic solid, the regime is linear and the solution is independent of $B i$. The corresponding values of $G^{\prime}$ and $G^{\prime \prime}$ are denoted by $G_{\infty}^{\prime}$ and $G_{\infty}^{\prime \prime}$. When $a= \pm 1$, from (18), we have

$$
G_{\infty}^{\prime}=\alpha / W e \quad \text { and } \quad G_{\infty}^{\prime \prime}=1-\alpha .
$$



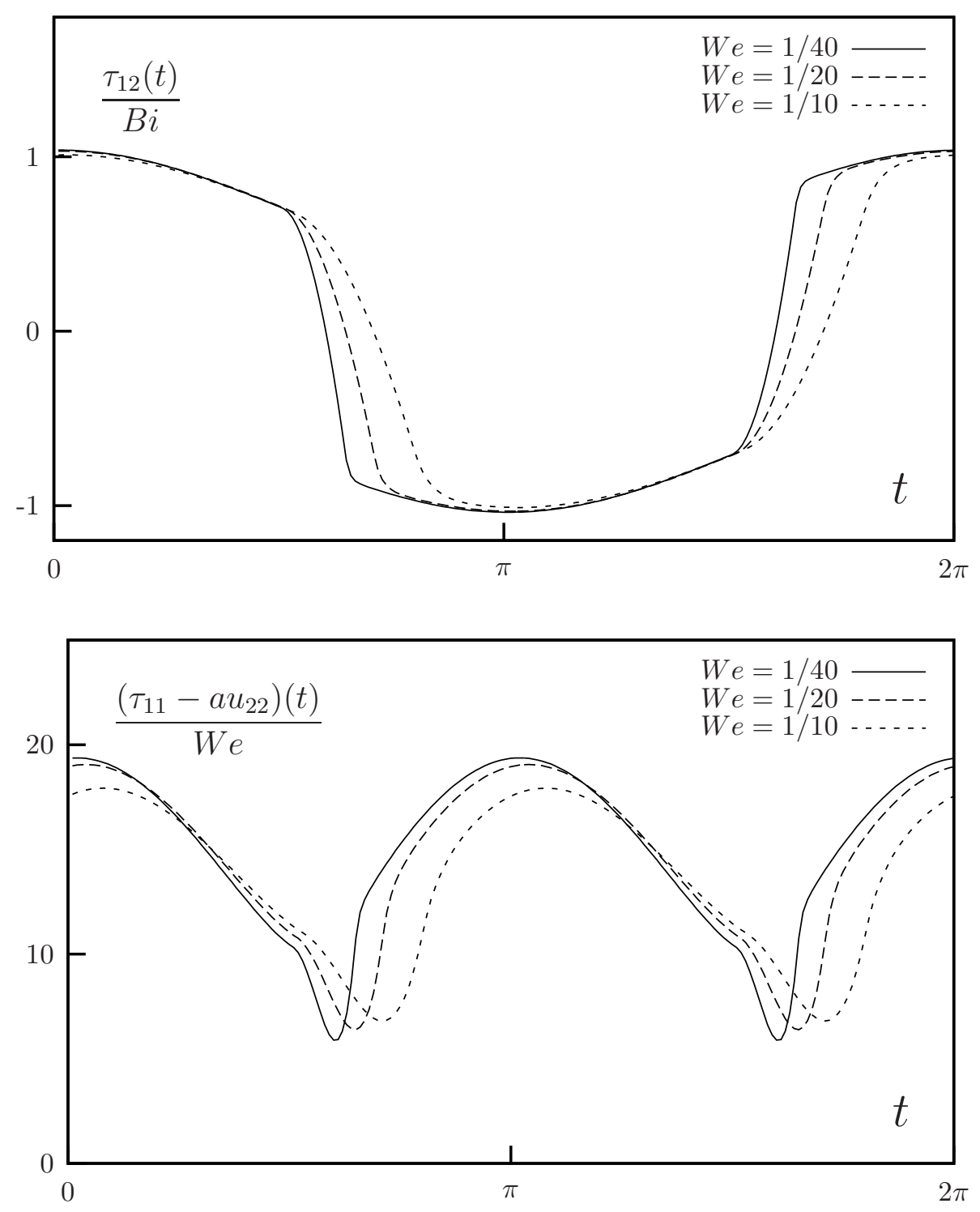

Figure 7. Periodic shear flow: influence of $W e=0.1,0.05,0.025$ : (top) $\tau_{12} / B i$; (bottom) $\tau_{11} / W e$.

Conversely, when $B i$ vanishes, the material behaves as a viscoelastic fluid: the constitutive equation is also linear and the solution is expressed by (16). The corresponding values of $G^{\prime}$ and $G^{\prime \prime}$ are denoted by $G_{0}^{\prime}$ and $G_{0}^{\prime \prime}$ :

$$
G_{0}^{\prime}=\frac{\alpha W e}{1+W e^{2}} \quad \text { and } \quad G_{0}^{\prime \prime}=1-\frac{\alpha W e^{2}}{1+W e^{2}}
$$

In the non-linear case, the moduli $\left(G^{\prime}, G^{\prime \prime}\right)$ are computed by using the solution $\left(\tau^{n}\right)_{0 \leq n \leq N}$ of the approximate problem (19): 

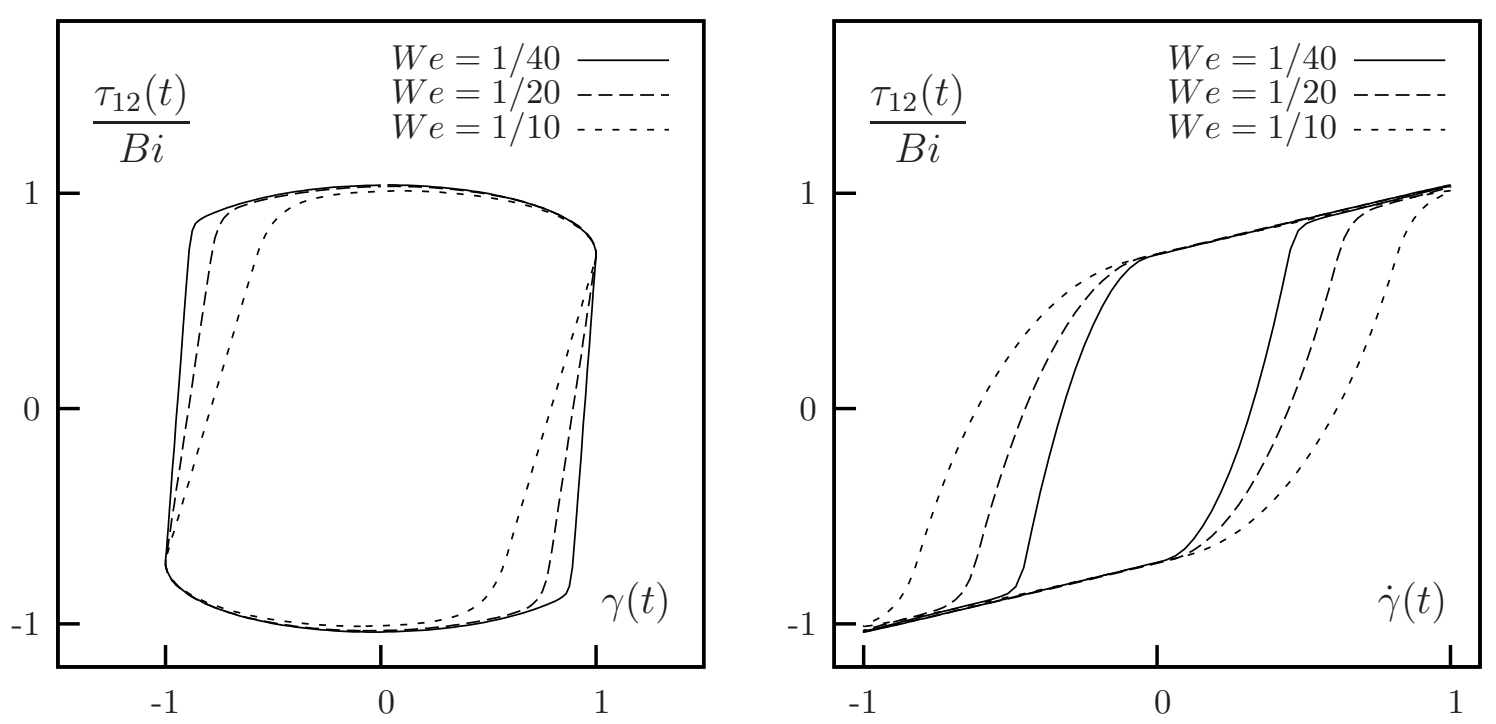

Figure 8. Lissajous plots: influence of $W e=0.1,0.05,0.025$ : (left) $\tau_{12} / B i$ versus $\gamma(t)=\sin (t)$; (right) $\tau_{12} / B i$ versus $\dot{\gamma}(t)=\cos (t)$.

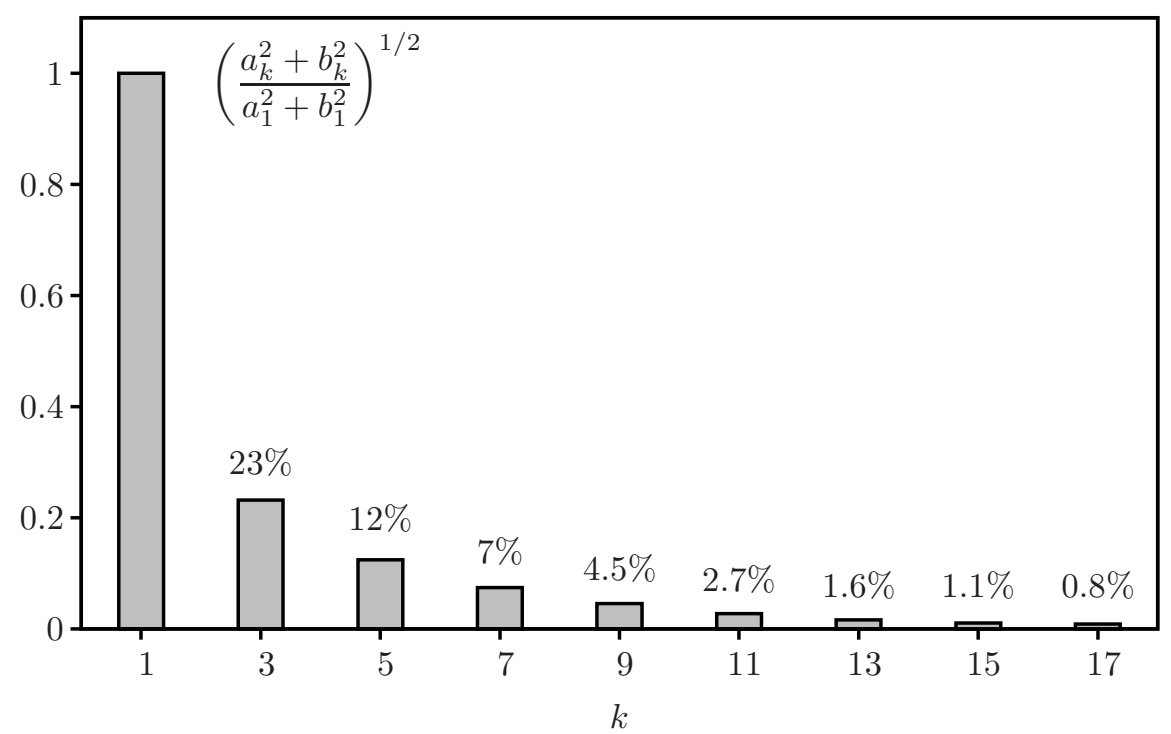

Figure 9. Periodic shear flow: intensities of the harmonics normalized by the intensity of the fundamental frequency: $W e=1 / 40, B i=3, a=1$ and $\alpha=8 / 9$.

$$
G^{\prime} \approx \frac{2}{N} \sum_{n=1}^{N} \tau_{12}^{n} \sin \left(t_{n}\right) \quad \text { and } \quad G^{\prime \prime} \approx 1-\alpha+\frac{2}{N} \sum_{n=1}^{N} \tau_{12}^{n} \cos \left(t_{n}\right) .
$$

The viscosity ratio parameter $\alpha=\eta_{m} /\left(\eta+\eta_{m}\right)$ controls $G_{\infty}^{\prime \prime}$, the $G^{\prime \prime}$ moduli at high values of $B i$ (i.e. for small amplitudes). The choice $\alpha<1$ leads to a non-vanishing $G_{\infty}^{\prime \prime}$, that has been found to be consistent 
with data measurements; the numerical computations are presented for $\alpha=8 / 9$.

Fig. 10 plots $G^{\prime}$ and $G^{\prime \prime}$ versus the dimensionless number $B i^{-1}=\gamma_{0} \eta_{0} \omega / \tau_{0}$. This is a dimensionless version of the moduli plots since most figures in the literature are based on the representation of the moduli versus the amplitude $\gamma_{0}$. Only $G^{\prime \prime}$ shows an overshoot: this behavior matches the type III of the
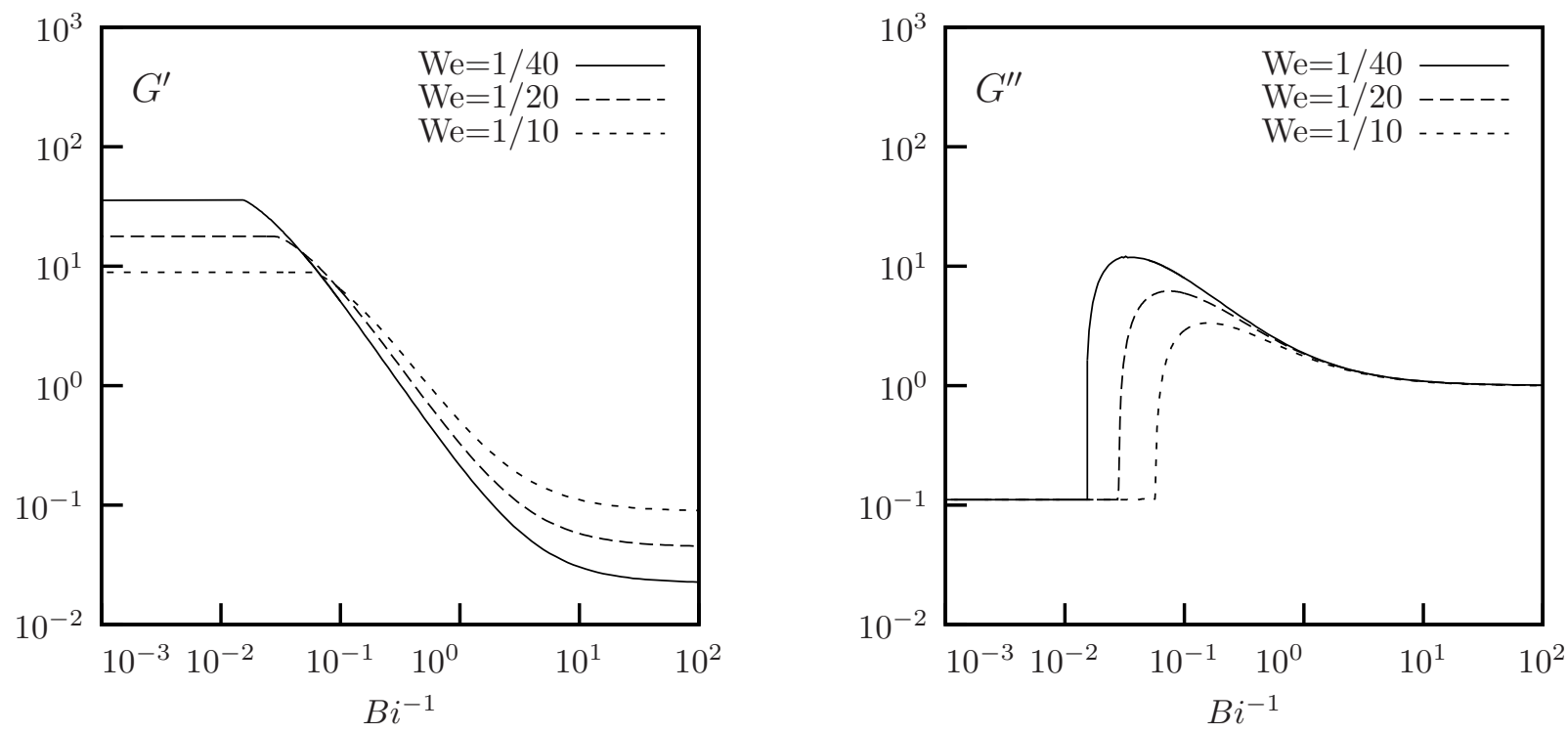

Figure 10. Periodic shear flow: moduli versus $B i^{-1}$ for $a=1$ : (left) $G^{\prime}$; (right) $G^{\prime \prime}$.

classification introduced in [8] when using large amplitude oscillatory shear. Many materials that present such a behavior are listed on page 61 of [8]. See also Fig. 7.b of this reference for the $\left(G^{\prime}, G^{\prime \prime}\right)$ plot of a Xanthan gum $4 \%$ solution. The micro-structure of this class of materials is characterized by a network of links that develops some complex structures. When an external strain is imposed, the complex network structure is destroyed by large deformation over a critical strain. In [21], based on a network model, the authors explain the overshoot of $G^{\prime \prime}$ in term of the balance between the formation and the destruction of the network junctions.

\section{Conclusion}

A new model for elastoviscoplastic fluid flows that is objective and satisfies the second law of thermodynamics is proposed in (11). A variant of the constitutive equation is also introduced in (12) in order to enforce finite extensional properties of the material. Large amplitude oscillatory shear has been performed and complex moduli $\left(G^{\prime}, G^{\prime \prime}\right)$ are qualitatively in good agreement with experimental data for many materials that present microscopic complex network structures with large rearrangements. The model is a good candidate for numerical simulation of elastoviscoplastic in multi-dimensional geometries: future works will perform such computations and compare them with experimental data measured on flows in complex geometries [2]. 


\section{Appendix A. Subdifferential calculus in the multi-dimensional case}

A.1. The $\varphi_{m}$ function - The subgradient $\partial \varphi_{m}$, as introduced in (7), is defined for any tensor $D$ by:

$$
\begin{aligned}
\partial \varphi_{m}(D) & =\left\{\tau, \tau:(H-D) \leq \varphi_{m}(H)-\varphi_{m}(D), \forall H\right\} \\
& =\left\{\tau, j_{m}(D) \leq j_{m}(H), \forall H \text { with } \operatorname{tr}(H)=0 \text { and } \operatorname{tr}(D)=0\right\},
\end{aligned}
$$

with the notation $j_{\tau}(H)=\eta_{m}|H|^{2}+\tau_{0}|H|-\tau: H$. When the minimizer $D$ of $j_{\tau}$ over the set $\{D, \operatorname{tr} D=0\}$ is non vanishing, it satisfies, from the theory of Lagrange multipliers:

$$
\nabla j_{\tau}(D)-p . I=0 \text { and } \operatorname{tr}(D)=0,
$$

where $p$ is the Lagrange multiplier. Then $2 \eta D+\tau_{0} \frac{D}{|D|}-\tau-p . I=0$ and $\operatorname{tr}(D)=0$. Thus the subgradient finally writes:

$$
\partial \varphi(D)= \begin{cases}\left\{\tau,\left|\tau_{d}\right| \leq \tau_{0}\right\} & \text { when } D=0, \\ \left\{\tau, \tau=-p . I+2 \eta_{m} D+\tau_{0} \frac{D}{|D|}\right\} & \text { when } D \neq 0 \text { and } \operatorname{tr}(D)=0, \\ \emptyset & \text { otherwise, }\end{cases}
$$

where $\tau_{d}$ denotes the deviatoric part of $\tau$. The dual $\varphi_{m}^{*}$ of $\varphi_{m}$ is then characterized by the Fenchel identity, that is, for any $\tau \in \partial \varphi_{m}(D)$, by $\varphi_{m}^{*}(\tau)=\tau: D-\varphi_{m}(D)$. Moreover, $\tau \in \partial \varphi_{m}(D)$ is equivalent to $D \in \partial \varphi_{m}^{*}(\tau)$. From $\tau+p . I=\left(2 \eta_{m}+\tau_{0} /|D|\right) D$ we get $\left|\tau_{d}\right|=2 \eta_{m}|D|+\tau_{0}$ and thus $|D|=\left(\left|\tau_{d}\right|-\tau_{0}\right) /\left(2 \eta_{m}\right)$. Finally:

$$
\partial \varphi_{m}^{*}(\tau)=\left\{D, D=\frac{1}{2 \eta_{m}} \max \left(0, \frac{\left|\tau_{d}\right|-\tau_{0}}{\left|\tau_{d}\right|}\right) \tau_{d}\right\}
$$

where $\tau_{d}$ denotes the deviatoric part of $\tau$.

A.2. The $\varphi$ function - The function $\varphi$, as introduced in (7), is a particular case of $\varphi_{m}$ with $\tau_{0}=0$ and $\eta_{m}=\eta$. From (A.1), the subgradient writes:

$$
\partial \varphi(D)= \begin{cases}\{\tau, \tau=-p . I+2 \eta D\} & \text { when } \operatorname{tr}(D)=0, \\ \emptyset & \text { otherwise. }\end{cases}
$$

\section{Appendix B. Explicit resolutions for one-dimensional creeping tests}

Let us suppose that the material is at the rest and that, for all $t \geq 0$ we impose a constant stress $\sigma(t)=\bar{\sigma}>0$. This appendix presents the explicit solution for three one-dimensional models: the present model, the Puzrin-Houlsby model and the Isayev-Fan model.

\section{B.1. The proposed model}

The potentials associated to the one-dimensional version of model presented on Fig. 1.c are:

$$
\mathcal{E}\left(\varepsilon, \varepsilon_{m}\right)=\frac{\mu}{2}\left|\varepsilon_{e}\right|^{2} \quad \text { and } \quad \mathcal{D}\left(\dot{\varepsilon}, \dot{\varepsilon}_{m}\right)=\frac{\eta}{2}|\dot{\varepsilon}|^{2}+\frac{\eta_{m}}{2}\left|\dot{\varepsilon}-\dot{\varepsilon}_{e}\right|^{2}+\tau_{0}\left|\dot{\varepsilon}-\dot{\varepsilon}_{e}\right| \text {. }
$$


By differentiation, as in (8), we obtain the constitutive law (5) with $\sigma=\eta \dot{\varepsilon}+\tau$. The imposed traction $\sigma(t)=\bar{\sigma}>0$ leads to $\dot{\varepsilon}=(\bar{\sigma}-\tau) / \eta$ and the constitutive equation (5) is then completed with the initial condition:

$$
\left\{\begin{aligned}
\lambda_{1} \frac{\mathrm{d} \tau}{\mathrm{d} t}(t)+\left(1+\frac{\eta}{\eta_{m}} \max \left(0, \frac{|\tau(t)|-\tau_{0}}{|\tau(t)|}\right)\right) \tau(t) & =\bar{\sigma} \quad \forall t>0, \\
\tau(0) & =0,
\end{aligned}\right.
$$

where $\lambda_{1}=\lambda \eta / \eta_{m}$. Since $\tau(0)=0$ and $\tau(t)$ is continuous there exists $t_{0}>0$ such that when $t \in\left[0, t_{0}\right]$ we have $|\tau(t)|<\tau_{0}$ : this is the first regime and the differential equation becomes linear: $\lambda_{1} \frac{\mathrm{d} \tau}{\mathrm{d} t}(t)+\tau(t)=\bar{\sigma}$, $\forall t \in] 0, t_{0}[$. Then

$$
\tau(t)=\bar{\sigma}\left(1-\exp \left(-t / \lambda_{1}\right)\right) \quad \text { and } \quad \varepsilon(t)=\left(\lambda_{1} \bar{\sigma} / \eta\right)\left(1-\exp \left(-t / \lambda_{1}\right)\right), \quad 0 \leq t \leq t_{0} .
$$

When $\bar{\sigma} \leq \tau_{0}$, we have always $\tau(t)<\tau_{0}$ and thus the previous expressions of $\tau$ and $\varepsilon$ are true for all $t \geq 0$. In that case, the elongation for a fixed imposed traction is bounded in time, which means that the material behaves as a solid. When $\bar{\sigma}>\tau_{0}$, we have $\tau\left(t_{0}\right)=\tau_{0}$ for a finite time $t_{0}=\lambda_{1} \log \left(\bar{\sigma} /\left(\bar{\sigma}-\tau_{0}\right)\right)$. When $t>t_{0}$ we have $\tau(t)>\tau_{0}$ : this is the second regime and the differential equation becomes: $\lambda_{2} \frac{\mathrm{d} \tau}{\mathrm{d} t}(t)+\tau(t)=\bar{\sigma}_{2}, \forall t>t_{0}$ together with the initial condition $\tau\left(t_{0}\right)=\tau_{0}$, where $\lambda_{2}=\lambda \eta /\left(\eta+\eta_{m}\right)$ and $\bar{\sigma}_{2}=\left(\eta_{m} \bar{\sigma}+\eta \tau_{0}\right) /\left(\eta+\eta_{m}\right)$. The solution in the second regime is:

$$
\begin{aligned}
& \tau(t)=\bar{\sigma}_{2}-\left(\bar{\sigma}_{2}-\tau_{0}\right) \exp \left(-\left(t-t_{0}\right) / \lambda_{2}\right), \quad t \geq t_{0}, \\
& \varepsilon(t)=\frac{\bar{\sigma}-\bar{\sigma}_{2}}{\eta}\left(t-t_{0}\right)+\frac{\lambda \tau_{0}}{\eta_{m}}+\frac{\lambda_{2}\left(\bar{\sigma}_{2}-\tau_{0}\right)}{\eta}\left(1-\exp \left(-\left(t-t_{0}\right) / \lambda_{2}\right)\right), \quad t \geq t_{0} .
\end{aligned}
$$

In that case, the elongation for a fixed imposed traction is unbounded in time, which means that such material behaves as a fluid. As a conclusion, the material behaves as an elastic solid when the traction is lower than the yield value and as a viscoelastic fluid otherwise (see Fig. 1.d).

\section{B.2. The Puzrin-Houlsby model}

The potentials associated to the one-dimensional version of model represented on Fig. 2.a are:

$$
\mathcal{E}\left(\varepsilon, \varepsilon_{m}\right)=\frac{\mu_{m}}{2}\left|\varepsilon_{m}\right|^{2}+\frac{\mu}{2}\left|\varepsilon-\varepsilon_{m}\right|^{2} \quad \text { and } \mathcal{D}\left(\dot{\varepsilon}, \dot{\varepsilon}_{m}\right)=f_{m}\left(\dot{\varepsilon}_{m}\right)
$$

where $f_{m}(d)=\tau_{0}|d|+\frac{\eta_{m}}{2}|d|^{2}, \forall d \in \mathbb{R}$. By differentiation, as in (8), we obtain the constitutive laws: $\sigma=\mu\left(\varepsilon-\varepsilon_{m}\right)$ and $\mu\left(\varepsilon-\varepsilon_{m}\right)-\mu_{m} \varepsilon_{m} \in \partial f_{m}\left(\dot{\varepsilon}_{m}\right)$. Since for all $\xi \in \mathbb{R}$ we have $\partial f_{m}^{*}(d)=\left\{\left(1 / \eta_{m}\right) \max \left(0,1-\tau_{0} /|d|\right) d\right\}, \forall d \in \mathbb{R}$, these relations can be rewritten as: $\varepsilon=\varepsilon_{m}+\sigma / \mu$ and

$$
\dot{\varepsilon}_{m}=\frac{1}{\eta_{m}} \max \left(0,1-\tau_{0} /\left|\sigma-\mu_{m} \varepsilon_{m}\right|\right)\left(\sigma-\mu_{m} \varepsilon_{m}\right) .
$$

For an imposed traction $\sigma=\bar{\sigma}$ and $\varepsilon_{m}(0)=0$ the problem is a nonlinear differential equation. When $\bar{\sigma} \leq \tau_{0}$ the solution is constant and otherwise the problem becomes linear. The solution for all $t>0$ is:

$$
\varepsilon(t)= \begin{cases}\bar{\sigma} / \mu & \text { when } \bar{\sigma} \leq \tau_{0}, \\ \bar{\sigma} / \mu+\frac{\bar{\sigma}-\tau_{0}}{\mu_{m}}(1-\exp (-t / \lambda)) & \text { otherwise. }\end{cases}
$$

As a conclusion, the Puzrin-Houlsby material behaves as an elastic solid when the traction is lower than the yield value and as a viscoelastic solid otherwise (see Fig. 2.b). 


\section{B.3. The one-mode simplified Isayev-Fan model}

The potentials associated to the one-dimensional version of model [9] represented on Fig. 2.c are:

$$
\begin{aligned}
& \mathcal{E}\left(\varepsilon, \varepsilon_{m, 1}, \varepsilon_{m, 2}\right)=\frac{\mu_{m}}{2}\left|\varepsilon_{m, 1}\right|^{2}+\frac{\mu}{2}\left|\varepsilon-\varepsilon_{m, 1}-\varepsilon_{m, 2}\right|^{2} \\
& \mathcal{D}\left(\dot{\varepsilon}, \dot{\varepsilon}_{m, 1}, \dot{\varepsilon}_{m, 2}\right)=f_{m}\left(\dot{\varepsilon}_{m, 1}+\dot{\varepsilon}_{m, 2}\right)+\frac{\eta_{m, 2}}{2}\left|\dot{\varepsilon}_{m, 2}\right|^{2}
\end{aligned}
$$

where $f_{m}(d)=\tau_{0}|d|+\frac{\eta_{m}}{2}|d|^{2}, \forall d \in \mathbb{R}$. The constitutive laws are obtained as usual by differentiation. Let $\sigma=\bar{\sigma}>0$ be the imposed traction and assume that $\varepsilon_{m, i}(0)=0, i=1,2$ as initial condition. The computation is comparable to the two previous examples and is not detailed here. When $\bar{\sigma} \leq \tau_{0}$ then the solution is constant: $\varepsilon(t)=\bar{\sigma} / \mu$, for all $t>0$. When $\bar{\sigma}>\tau_{0}$, after reduction to some linear problems, we obtain the solution for all $t>0$ :

$$
\varepsilon(t)= \begin{cases}\bar{\sigma} / \mu & \text { when } \bar{\sigma} \leq \tau_{0}, \\ \bar{\sigma} / \mu+b t+(c-b \lambda)(1-\exp (-t / \lambda)) & \text { otherwise }\end{cases}
$$

where

$$
\lambda=\left(\frac{\eta_{m} \eta_{m, 2}}{\eta_{m}+\eta_{m, 2}}\right) \mu_{m}, \quad b=\frac{\bar{\sigma}-\tau_{0}}{\eta_{m}+\eta_{m, 2}} \quad \text { and } \quad c=\left(\frac{\eta_{m} \eta_{m, 2}}{\eta_{m}+\eta_{m, 2}}\right) \frac{\bar{\sigma}-\tau_{0}}{\mu_{m}} .
$$

As a conclusion, the Isayev-Fan simplified material behaves as an elastic solid when the traction is lower than the yield value and as a viscoelastic fluid otherwise (see Fig. 2.d). 


\section{References}

[1] E. C. Bingham. Fluidity and plasticity. Mc Graw-Hill, 1922.

[2] B. Dollet, M. Durth, F. Graner. Flow of a foam past an elliptical obstacle. Phys. Rev. E, 73, 2006.

[3] D. Doraiswamy, A. N. Mujumdar, I. Tsao, A. N. Beris, S. C. Danforth, A. B. Metzner. The Cox-Merz rule extended: a rheological model for concentrated suspensions and other materials with a yield stress. J. Rheol., 35(4):647-685, 1991.

[4] R. J. Gordon, W. R. Schowalter. Anisotropic fluid theory: a different approach to the dumbbell theory of dilute polymer solutions. Trans. Soc. Rheol., 16:79-97, 1972.

[5] B. Halphen, Q. S. NGuyen. Sur les matériaux standard généralisés. J. Méca., 14:39-63, 1975.

[6] W. H. Herschel, T. Bulkley. Measurement of consistency as applied to rubber-benzene solutions. Am. Soc. Test Proc., 26(2):621-633, 1926.

[7] G. T. Houlsby, A. M. Puzrin. Rate-dependent plasticity models derived from potential functions. J. Rheol., 46(1):113126, 2002.

[8] K. Hyun, S. H. Kim, K. H. Ahn, S. J. Lee. Large amplitude oscillatory shear as a way to classify the complex fluids. J. Non-Newtonian Fluid Mech., 107:51-65, 2002.

[9] A. Isayev, X. Fan. Viscoelastic plastic constitutive equation for flow of particle filled polymers. J. Rheol., 34(1):35-54, 1990.

[10] G. Maugin. The thermomechanics of plasticity and fracture. Cambridge University Press, 1992.

[11] J. Oldroyd. A rational formulation of the equations of plastic flow for a Bingham solid. Proc. Camb. Philos. Soc., 43:100-105, 1947.

[12] J. G. Oldroyd. On the formulation of rheological equations of states. Proc. Roy. Soc. London, A 200:523-541, 1950.

[13] N. Phan-Thien, R. I. Tanner. A new constitutive equation derived from network theory. J. Non-Newtonian Fluid Mech., 2:353-365, 1977.

[14] W. Prager, K. Hohenemser. Über die Ansätze der Mechanik isotroper Kontinua. Z. Angew. Math. Mech., 12:216-226, 1932.

[15] A. Puzrin, G. Houlsby. A thermomechanical framework for rate-independent dissipative materials with internal functions. Int. J. Plast., 17(8):1147-1165, 2001.

[16] A. Puzrin, G. Houlsby. Rate-dependent hyperplasticity with internal functions. J. Engrg. Mech., 51(3):252-263, 2003.

[17] N. Roquet, P. Saramito. An adaptive finite element method for Bingham fluid flows around a cylinder. Comput. Appl. Meth. Mech. Engrg., 192(31-32):3317-3341, 2003.

[18] P. Saramito. Operator splitting for viscoelastic fluid with a differential constitutive law. C. R. Acad. Sci. Paris, Série II, t. 319, No. 3:267-270, 1994.

[19] P. Saramito. Efficient simulation of nonlinear viscoelastic fluid flows. J. Non-Newtonian Fluid Mech., 60:199-223, 1995.

[20] T. Schwedoff. La rigidité des fluides. In Rapports du Congrès Intern. de Physique, volume 1, pages 478-486. 1900.

[21] H. G. Sim, K. H. Ahn, S. J. Lee. Large amplitude oscillatory shear behavior of complex fluids investigated by a network model: a guideline for classification. J. Non-Newtonian Fluid Mech., 112:237-250, 2003.

[22] P. L. Tallec. Numerical analysis of viscoelastic problems. Masson, France, 1990.

[23] D. Vlassopoulos, S. G. Hatzikiriakos. A generalized Giesekus constitutive model with retardation time and its association to the spurt effect. J. Non-Newtonian Fluid Mech., 57(2-3):119-136, 1995.

[24] R. von Mises. Mechanik der festen Körper im plastich-deformablen Zustand. Nachrichten Ges. Wiss. Göttingen, pages 582-592, 1913.

[25] P. Wapperom, A. Leygue, R. Keunings. Numerical simulation of large amplitude oscillatory shear of a high-density polyethylene melt using the MSF model. J. Non-Newtonian Fluid Mech., 130:63-76, 2005. 